\title{
Investigating Positive and Negative Affects in Collaborative Information Seeking: A Pilot Study Report
}

\author{
Roberto González-lbáñez ${ }^{182}$, Chirag Shah ${ }^{1}$ \\ ${ }^{1}$ School of Communication \& Information, Rutgers University, 4 Huntington St, New Brunswick, NJ 08901 \\ ${ }^{2}$ Departamento de Ingeniería Informática, Universidad de Santiago de Chile, Avenida Ecuador 3659, Estación \\ Central, Santiago, Chile \\ \{rgonzal, chirags\}@rutgers.edu
}

\begin{abstract}
Emotions and other affective processes have long been considered a key component in people's life. Despite research conducted in several research domains, little is known about the role of emotions in the information seeking process of both individuals and teams. This poster presents preliminary results from a pilot study conducted in order to evaluate design decisions, experimental protocol, tasks, and the system that will be used in research aiming to investigate the implications of positive and negative affects in the information search process of both individuals and teams. The pilot study involved 12 subjects randomly assigned to five experimental conditions in which a common information search task was performed. In each session, facial expressions, eye tracking data, electrodermal activity, desktop activity, users' actions, and searches as well as communication logs were collected. In addition, as sessions were conducted, observations about subjects' behaviors, system problems, and research protocols were made. Results from this pilot study suggest effects of both positive and negative affects at the level of performance, communication, and perceived difficulty, among other aspects. In addition to these preliminary findings, the poster also contributes a unique methodology, involving a rigorous design, for conducting such user studies.
\end{abstract}

\section{Keywords}

Collaborative search, interactive search, affects. INTRODUCTION

It has been argued that emotions play an important role in different aspects of life such as survival or communication. For example, studies in psychology and economics, among other disciplines have shown that human emotions are necessary for decision making [3], a process that has long been attributed to rational thinking.

Although emotions have been widely studied in different research domains, little is known about their participation in the information search process (ISP) of both individuals and teams. Some researchers have indicated that emotions are present at different stages of the ISP [11]; however, their implications on the way people search or assess information

Copyright held by the author(s)

Proceedings of HCIR 2012, Cambridge MA, Oct 4-5, 2012 are still understudied. Recent studies have partially addressed research questions about the emotions that individuals would experience during the ISP and how they would relate to specific search behaviors [7][12].

In collaborative scenarios, studies in this regard are scarcer. In collaborative information seeking (CIS), for example, emotions have been acknowledged to take an active part in the ISP of teams [10]; nevertheless, to the best of our knowledge, no studies have been specially designed to investigate emotions in this domain.

This poster presents a pilot study conducted as part of a major research plan aimed at understanding what role emotions and other affective processes play in the ISP of both individuals and teams. Unlike previous studies, this research was specifically designed to take a closer look at different affective processes that take place within the ISP.

\section{BACKGROUD}

We begin by introducing definitions of two key concepts used in this study: emotion and CIS. First of all, we adopted Palmero et al.'s [13] definition of emotions and related concepts such as mood, affect, and feeling. In summary, the term emotion refers to spontaneous and short-duration affective responses that can be classified under a categorical approach (e.g. happy, sad, angry). The terms affect and mood, though slightly different, correspond to affective states that last longer than emotions and are described under a dimensional approach (e.g. arousal: excited-calm, valence: positive - negative). Finally, feelings correspond to the subjective component of underlying affective processes. In other words, feelings take place when individuals become aware that they are experiencing a given emotion or mood. The second concept corresponds to CIS, which according to Foster [5] corresponds to "the study of the systems and practices that enable individuals to collaborate during the seeking, searching, and retrieval of information" (p. 330).

In information science, Kuhlthau [11] introduced one of the first models that considered feelings as an active part of the ISP of individuals. More recently, the introduction of new technologies and methodologies have enabled scientists to take a closer look at the problem of the affective dimension in information science, enabling them to quantitatively measure affective factors in the ISP. For example, Lopatovska [12] and González-Ibáñez et al. [7] 
incorporated automatic facial expression recognition as well as self-assessment to study the expressivity of basic emotions and the experience of feelings in an information search task.

In CIS, however, emotions are still understudied. Hyldegard [10] and Shah and González-Ibáñez [15], for example, explored the applicability of Kulhtauh's ISP model to collaborative settings, with a limited attention given to exploring emotions in the ISP of teams. González-Ibáñez et al. [7] studied the expressivity of happiness in the ISP of pairs working in an exploratory search task. According to the authors, unlike individual information seeking, in collaborative settings team members are more likely to express emotions. However, such episodes occur as a result of interactions among team members and they are not necessarily connected to information-related events.

Regardless of the differences between individuals and teams, emotions could be not only a consequence but also a cause of information behaviors. As pointed out in [7], the following two questions should be addressed: "[(Q1)] do [people] feel a certain way because [they] find (or do not find) information? or [(Q2)] do [people] find (or do not find) information because [they] feel a certain way?" (p. 2). While emotion research in information seeking has focused primarily in Q1, the implications of prior affective processes in the search process (Q2) of both individuals and teams may have larger implications in this research field. From a positive-psychology perspective, both positive and negative affects would have a crucial role in daily activities [4]. If emotions play such a role in people's life, would it be the case that they also change the way people search, assess, and make sense of information?

The study introduced in this poster aims to investigate both questions. It is important to note, however, that the pilot study described in the next section was not intended to fully address these questions, but rather, to assess design decisions, experimental protocol, task, stimuli, and the experimental system. This may limit the scope of the results presented in this poster.

\section{PILOT STUDY}

This section introduces key aspects of a pilot study conducted in order to evaluate design decisions, experimental protocol, task, and the system that will be used in research aiming to investigate the implications of positive and negative affects in the information search process of both individuals and teams.

\section{Participants}

Participants in this pilot study were 12 undergraduate students from Rutgers University. As explained below, this study involved two individual conditions and three collaborative conditions. For the individual conditions we recruited six participants. For the collaborative condition, we recruited six participants in three pairs. Individuals and pairs were randomly assigned to the corresponding experimental conditions. In order to ensure common ground among team members [2], in the latter case participants signed up in pairs with someone with whom they had previous experience working together. Participants were required to be native English speakers with intermediate typing and online search skills, and be 18 to 24 years old. Participants in this study received extra credit for a class or were paid $\$ 10$ in cash. Moreover, regardless of the experimental condition to which participants were assigned, subjects were informed in advance of the possibility of winning cash prizes if they were amongst the best three performing participants or teams.

\section{Experimental Conditions}

This pilot study involved five experimental conditions, each one related to treatments to elicit positive and negative affects in participants. As listed below, three of these conditions involved pairs of individuals who collaborated synchronously and remotely located using text-chat to communicate:

$\mathbf{X}_{++}$: Both team members receive stimuli to elicit positive affects prior to starting the task.

$\mathbf{X}_{+.}$: One team member receives stimuli to elicit positive affects and the other stimuli to elicit negative affects prior to starting the task.

$\mathbf{X}_{\text {..: }}$ Both team members receive stimuli to elicit negative affects prior to starting the task.

The other two conditions focused on individual information seeking and at the same time they constitute a baseline for comparison. In the first of these conditions $\left(\mathrm{X}_{+}\right)$, subjects receive stimuli to elicit positive affects prior to starting the task. In the second one (X.), subjects receive stimuli to elicit negative affects prior to starting the task.

\section{Experimental Design}

\begin{tabular}{cccccc}
\hline $\mathrm{R}$ & $\mathrm{O}_{1}$ & $\mathbf{X}_{++}$ & $\mathrm{O}_{\mathrm{n}}$ & $\mathrm{T}$ & $\mathrm{O}_{\mathrm{n}+\mathrm{m}}$ \\
$\mathrm{R}$ & $\mathrm{O}_{1}$ & $\mathbf{X}_{+-}$ & $\mathrm{O}_{\mathrm{n}}$ & $\mathrm{T}$ & $\mathrm{O}_{\mathrm{n}+\mathrm{m}}$ \\
$\mathrm{R}$ & $\mathrm{O}_{1}$ & $\mathbf{X}_{-.}$ & $\mathrm{On}_{\mathrm{n}}$ & $\mathrm{T}$ & $\mathrm{O}_{\mathrm{n}+\mathrm{m}}$ \\
$\mathrm{R}$ & $\mathrm{O}_{1}$ & $\mathbf{X}_{+}$ & $\mathrm{O}_{\mathrm{n}}$ & $\mathrm{T}$ & $\mathrm{O}_{\mathrm{n}+\mathrm{m}}$ \\
$\mathrm{R}$ & $\mathrm{O}_{1}$ & $\mathbf{X}_{.}$ & $\mathrm{O}_{\mathrm{n}}$ & $\mathrm{T}$ & $\mathrm{O}_{\mathrm{n}+\mathrm{m}}$ \\
\hline
\end{tabular}

Table 1. Between subjects design. (R): Randomization, (O): observations, $(X)$ : treatment, and $(T)$ : task.

As depicted in Table 1, the five conditions were incorporated in a between subjects design that was specially devised to evaluate affective processes in the ISP of both individuals and teams. In terms of treatments (X), among the variety of possible stimuli that can be applied to elicit specific affective responses in each condition, we chose false-feedback [16]. This technique consists on providing either positive (e.g. "you are doing great!) or negative (e.g. "Wrong. That was disappointing") feedback to the user regardless of his/her actual performance when working on a given task. The duration of stimuli stage was set in seven minutes. Following the stimuli stage, participants had to perform the main task. During the entire session, both discrete and continuous observations (O) were performed. The overall experimental design is depicted in Table 1.

\section{Task Description}

A multiple-step fact-finding and non-dividable search task was designed for this study. The task consisted of answering questions randomly collected from A Google a 
Day (http://agoogleaday.com). Each question was objectively rated based on the number of steps or queries suggested in A Google a Day to find its answer. As a result, the number of steps (complexity level) for the entire set of questions ranged between 2 and 5. For this study, only level-2 and level-3 questions were presented to the subjects alternately. For each question participants were given up to five minutes to respond. While the task is not intended to be realistic, it is adequate to properly investigate and evaluate affective reactions and their implications in the ISP in experimental settings, which is the focus of this research.

\section{Instruments, Systems, and Research Methods}

In order to collect both quantitative and qualitative data, different systems and instruments were used. At the hardware level we used Affectiva Q-Sensors 2.0 to measure electrodermal activity (EDA), which is known to be related to affective responses. Web cameras were used to capture participants' facial expressions. Mirametrix S2 Eyetrackers were used to capture participants' eye fixations. In terms of software, different logging tools such as Coagmento Collaboratory - an adapted version of Coagmento [6][14] and TechSmith Morae 3.1 were used to record and monitor users' actions. Additionally, different questionnaires were used to perform observations before, during, and after the task. Some of the questionnaires we used are: SAM [1] to measure pleasure, arousal, and dominance; and NASA-TLX [9] for cognitive load. Finally, a semi-structured interview was conducted at the end of each session.

\section{PRELIMINAY RESULTS}

This section presents an overview of the results obtained in the pilot study. The main source of data analyzed were subjects' search logs and responses to questionnaires. Results are based mainly on means and percentages. Analyses of significance were not performed due to the sample size.

Analyses of the responses to the questionnaire about topic familiarity and perceived complexity (both questions responded at the moment of reading each question for the first time) showed that participants were not familiar at all or barely familiar with the majority of the questions (77\%). A more detailed view revealed that participants were more familiar with the topics of level-3 questions than those of level-2 questions. In terms of perceived complexity, participants indicated $47.92 \%$ of the time that level-2 questions were very complex to address. Conversely, for level-3 questions that perception falls to $33.33 \%$. Looking at the responses of subjects based on the experimental conditions, it was found that those who were treated with negative stimuli reported being less familiar with the topic of questions than those who received positive stimuli; this was reflected in $24.32 \%$ more responses. Similarly, those who received negative stimuli rated questions as very complex in twice as many answers than those who received positive stimuli. At the moment of providing the answer to the question, participants reported that level-3 questions were more difficult to address than level-2 questions (50\% and $26 \%$ of the responses respectively). Consequently, when asked about their level of confidence with respect to the answer provided to each question, it was higher for level-2 questions than level-3 questions. In terms of experimental conditions, participants who were treated with negative stimuli found that questions were very difficult. The latter was indicated in $12.5 \%$ more responses than those provided by participants who received positive stimuli. Looking at the response time per experimental condition, results show that participants who received negative stimuli tended to spend less time in each question than those who were in positive conditions. In the condition in which both participants received positive stimuli $\left(\mathrm{X}_{++}\right)$, the average response time per question was almost twice the time spent by teams in which both subjects were treated with negative stimuli ( $\left.\mathrm{X}_{-}\right)$.

An evaluation of time per question with respect to each level revealed that participants spent more time working on level-2 questions (mean=3.43, s.d.=1.57) than on level-3 questions (mean=2.496, s.d=1.382).

Each answer was classified in one of the following four categories: (1) incorrect (2) correct (3) partially correct, if the answer contained part of the elements required in the full answer (e.g. in a list of three names, only two were provided); and (4) pieces of information, if the answer provided was in the search path that leads to find the correct answer (e.g. the right answer was a specific town in a city, but subjects provided the name of the city). Since answers were freely entered in an empty text field and thus subject to typos, the evaluation of answers was manually performed. Results showed that most answers were incorrect (68.235\%) and only $15.294 \%$ were completely correct. The remaining $16.471 \%$ of the answers were evaluated as partially correct and pieces of information ( $8.235 \%$ each). In terms of the level of questions, subjects found twice as many right answers for level-2 questions than for level-3 questions. An analysis per experimental condition indicates that those in which participants received positive stimuli reached slightly better results in terms of the precision of their answers (ratio between right answers over the total number of questions addressed during the session) than those who received negative stimuli.

Each question involves a different search process with one or multiple search trails. For this pilot study a simple analyses was performed in order to investigate possible differences among conditions. The first analysis conducted was the evaluation of the direct implication of the positive and negative stimuli in non-collaborative conditions when working on the very first question after the stimuli stage. A simple comparison indicates that participants who received positive stimuli formulated more queries (mean $=9.667$, s.d.=2.081) than those who received negative stimuli (mean=9.0, s.d.=7.0). Conversely, those in the negative condition visited slightly more pages (mean=4.667, s.d.=3.786) than those in the positive condition (mean=4.0, s.d.=1.0). In terms of search result pages (SERPs), participants in the positive condition were exposed to more SERPs $($ mean=12.667, s.d.=4.041) than those in the 
negative condition (mean=10.0, s.d.=7.211). The data collection process resulted in more snippets saved by participants in the negative condition (mean $=2.5$, s.d.=2.121), than those in the positive condition (mean=1.0, s.d.=1.0). Finally, the time spent (in minutes) in the first question was higher for those working in the positive condition (mean=5.076, s.d.=0.718) than those in the negative condition ( mean $=3.862$, s.d. $=0.903$ ).

For collaborative conditions, a quick communication analysis in terms of the number of messages exchanged in each question and also per condition was performed. We found that the volume of messages exchanged tends to decrease as sessions progress. Based on observations performed during the experiment, it was found that messages were task oriented [8]. Even though the task was non-dividable, participants had independency to work in each question and communication took place when decisions had to be made. An analysis per condition indicates that the volume of communication was higher in the mixed affect condition (+-). While the difference is smaller compared to the positive condition (++), it was found twice as many messages exchanged in the mixed condition than in the negative one (--).

\section{DISCUSSION AND NEXT STEPS}

The preliminary results presented in the above section indicate that both positive and negative affect would have potential implications to the search process of individuals and teams. In addition to these results, this pilot study allowed us to assess the design as well as other components of the proposed study. As a result of this evaluation, the experimental design was updated by pre-task and post-task stages in each condition. Moreover, a control condition was added in order to properly evaluate if the different stimuli applied to subjects are actually responsible for producing the variations in the ISP. The evaluation performed right after treatments are applied is denominated in this new design the short-term effect (10 minutes). On the other hand, the main task evaluation aims to study how positive and negative stimuli change the way people search information individually or with others during the following 25 minutes (prolonged effect) (Table 2).

\begin{tabular}{llllllllll}
\hline $\mathrm{R}$ & $\mathrm{O}_{1}$ & PreT & $\mathrm{O}_{2}$ & $\mathbf{X}++$ & $\mathrm{O}_{\mathrm{n}}$ & PosT & $\mathrm{O}_{\mathrm{n}+1}$ & MT & $\mathrm{O}_{\mathrm{n}+\mathrm{m}}$ \\
$\mathrm{R}$ & $\mathrm{O}_{1}$ & PreT & $\mathrm{O}_{2}$ & $\mathbf{X}+-$ & $\mathrm{O}_{\mathrm{n}}$ & PosT & $\mathrm{O}_{\mathrm{n}+1}$ & MT & $\mathrm{O}_{\mathrm{n}+\mathrm{m}}$ \\
$\mathrm{R}$ & $\mathrm{O}_{1}$ & PreT & $\mathrm{O}_{2}$ & $\mathbf{X}-$. & $\mathrm{O}_{\mathrm{n}}$ & PosT & $\mathrm{O}_{\mathrm{n}+1}$ & MT & $\mathrm{O}_{\mathrm{n}+\mathrm{m}}$ \\
$\mathrm{R}$ & $\mathrm{O}_{1}$ & PreT & $\mathrm{O}_{2}$ & $\mathbf{X}+$ & $\mathrm{O}_{\mathrm{n}}$ & PosT & $\mathrm{O}_{\mathrm{n}+1}$ & MT & $\mathrm{O}_{\mathrm{n}+\mathrm{m}}$ \\
$\mathrm{R}$ & $\mathrm{O}_{1}$ & PreT & $\mathrm{O}_{2}$ & $\mathbf{X}-$ & $\mathrm{O}_{\mathrm{n}}$ & PosT & $\mathrm{O}_{\mathrm{n}+1}$ & MT & $\mathrm{O}_{\mathrm{n}+\mathrm{m}}$ \\
$\mathrm{R}$ & $\mathrm{O}_{1}$ & PreT & $\mathrm{O}_{2}$ & & $\mathrm{O}_{\mathrm{n}}$ & PosT & $\mathrm{O}_{\mathrm{n}+1}$ & MT & $\mathrm{O}_{\mathrm{n}+\mathrm{m}}$ \\
\hline
\end{tabular}

Table 2. New experimental design summary. (R): Randomization, (PreT): Pre-Task, (PosT): Post-Task, (O): observations, $(X)$ : treatment/stimuli, and (MT): main task.

With this new experimental design, hypotheses such as the following can be tested:

$\mathrm{H} 1$ : Participants who receive negative stimuli will reach a lower quality of work (response precision) than those who are positively treated.

H2: Participants who receive negative stimuli and collaborate with someone who was also treated with negative stimuli will reach a lower quality of work (response precision) than teams in which one or two members were positively treated.

In our future work we will address the problem of affective dimension in CIS following the experimental design presented in Table 2. Our next step is to proceed with a new evaluation of the changes introduced as a result of the evaluation of this pilot study. Then, the main study will be conducted.

\section{REFERENCES}

1. Bradley, M. M., and Lang, P. J. (1994). Measuring Emotion: The SelfAssessment Manikin and the Semantic Differential. Journal of Behavior Therapy and Experimental Psychiatry. 22(1): 49-59.

2. Clark, H. H, and Brennanm S. E. (1991). Grounding in communication. In L. B. Resnick, R. M. Levine, and S. D. Teasley (Eds.), Perspectives on socially shared cognition (pp. 127-149). The American Psychological Association.

3. Figner, B., and Murphy, R. O. (2011). Using skin conductance in judgment and decision making research. In M. Schulte-Mecklenbeck, A. Kuehberger, and R. Ranyard (Eds.), A handbook of process tracing methods for decision research (pp. 163-184). New York, NY: Psychology Press.

4. Fredrickson, B. L. and Losada, M. F. (2005). The Positive Affect and the Complex Dynamics of Human Flourishing. American Psychologist, 60(7), 678-686.

5. Foster, J. (2006). Collaborative information seeking and retrieval. Annual Review of Information Science and Technology (ARIST), 40, 329-356.

6. González-Ibáñez, R., and Shah, C. (2011). Coagmento: A System for Supporting Collaborative Information Seeking. Integrated demo at ASIST'2011, New Orleans, LA.

7. González- Ibáñez, R., Shah, C., and Córdova-Rubio, N. (2011). Smile! Studying Expressivity of Happiness as a Synergic Factor in Collaborative Information Seeking. ASIST 2011, New Orleans, LI.

8. González-Ibáñez, R., Haseki, M., and Shah, C. (2012). Understanding Effects of Time and Proximity on Collaboration: Implications for Technologies to Support Collaborative Information Seeking. Work in progress to be presented at $\mathrm{CHI}^{\prime} 12$, Austin, TX.

9. Hart, S. G., and Staveland, L. E. (1988) Development of NASA-TLX (Task Load Index): Results of empirical and theoretical research. In P. A. Hancock and N. Meshkati (Eds.) Human Mental Workload. Amsterdam: North Holland Press.

10. Hyldegard, J. (2006) Collaborative Information Behaviour - Exploring Kuhlthau's Information Search Process Model in a Group-based Educational Setting. Information Processing and Management, 42(1), 276-298.

11. Kuhlthau, C. (1991). Inside the Search Process: information seeking from the user's perspective. Journal of the American Society for Information Science, 42 (6), 361-371.

12. Lopatovska, I. (2009). Does the mood matter?. Proceedings of the 2009 International Conference on Affective Computing \& Intelligent Interaction ACII 2009, pp. 237-240, Amsterdam, The Netherlands.

13. Palmero, F., Guerrero, C., Gómez, C., and Carpi, A. (2006). Certezas y controversia en el estudio de la emoción. Revista electrónica de motivación y emoción (R.E.M.E), 9, 23-24.

14. Shah, C. (2010). Coagmento - A Collaborative Information Seeking, Synthesis and Sense-Making Framework. Integrated demo at CSCW 2010. Savannah, GA: February 6-11, 2010.

15. Shah, C., and González-Ibáñez, R. (2010). Exploring Information Seeking Processes in Collaborative Search Tasks. In Proceedings of American Society of Information Science and Technology (ASIST). Pittsburgh, PA. October 22-27, 2010.

16. Zhao, J. (2006). The Effects of Induced Positive and Negative Emotions on Risky Decision Making. Talk presented at the 28th Annual Psychological Society of Ireland Student Congress, Maynooth, Ireland. 